\title{
As estratégias de divulgação científica e transferência de tecnologia utilizada pela Empresa Brasileira de Pesquisa Agropecuária (Embrapa)
}

Strategies for scientific dissemination and transfer of technology used by the Empresa Brasileira de Pesquisa Agropecuária (Embrapa)

\section{Stratégies pour La diffusion scientifique et letransfert de latechnologie utilisée par Empresa Brasileira de Pesquisa Agropecuária (Embrapa)}

Las estrategias para La divulgación científica y La transferencia de La tecnología utilizada por la Empresa Brasileira de Pesquisa Agropecuária (Embrapa)

\author{
Nádia Solange Schmidt Bassi* \\ (nadia.bassi@embrapa.br) \\ Christian Luiz da Silva** \\ (christiansilva@utfpr.edu.br)
}

Recebido em 10/12/2012; revisado e aprovado em 08/12/2012; aceito em 03/02/2013

\begin{abstract}
Resumo: O objetivo deste artigo é descrever e analisar os processos de comunicação científica e transferência de tecnologia da Empresa Brasileira de Pesquisa Agropecuária (Embrapa), e de sua unidade de pesquisa denominada Embrapa Suínos e Aves, com a proposição, ao final, da criação de novos modelos de comunicação e de transferência de tecnologia, considerando a diversidade de seu público-alvo e a natureza de suas atividades.
\end{abstract}

Palavras-chave: Comunicação científica. Transferência de tecnologia. Embrapa.

Abstract: The aim of this study is to describe and analyze the process of scientific and technology transfer from the Empresa Brasileira de Pesquisa Agropecuária (Embrapa), and its research unit called Embrapa Swine and Poultry, with the proposition, at the end, of the creation of new models communication and technology transfer, considering the diversity of your target audience and the nature of its activities.

Key words: Scientific communication. Technology transfer. Embrapa.

Résumé: Le but de cet article est de décrire et d'analyser les processus de communication scientifique et le transfert de technologie de Empresa Brasileira de Pesquisa Agropecuária (Embrapa) et son unité de recherche appelé Embrapa porcs et lavolaille, avec la proposition à la fin, la création de nouveaux modèles de communication et le transfert de technologie, en prenant en compte la diversité de votre public cible et la nature de ses activités.

Mots-clés: La communication scientifique. Transfert de technologie. Embrapa.

Resumen: El propósito de este artículo es describir y analizar los procesos de comunicación científica y transferencia de tecnología de la Empresa Brasileira de Pesquisa Agropecuária (Embrapa) y su unidad de investigación llaman Embrapa porcinos y aves de corral, com la propuesta hasta el final, la creación de nuevos modelos de comunicación y la transferencia de tecnología, teniendo en cuenta la diversidad de su público objetivo y la naturaleza de sus atividades. Palabras clave: Comunicación científica. Transferencia de tecnología. Embrapa.

\section{Introdução}

As instituições públicas que atuam na área de Ciência e Tecnologia (C\&T) são atores sociais mantidos pela sociedade e têm a responsabilidade de prestar contas do uso dos recursos e contribuir para a popularização da ciência. Para Geller (2010), é fundamental que os temas científicos sejam amplamente divulgados, para que os cidadãos tenham elementos para opinar e influenciar nas situações que afetam suas vidas. Segundo Wildner et al. (1993) e Araújo (1979), a transferência inadequada pode ser resultante de uma co- municação deficiente entre a instituição e os usuários. Os autores observaram também que a tecnologia inapropriada é mais comum do que a transferência inadequada como causa de sua baixa adoção pelos usuários. Essa opinião é corroborada por Fujisaka (1994), que identificou que uma das razões pelas quais usuários não adotam tecnologias é que esta resulta de um problema mal definido pela pesquisa, isto é, os usuários não enfrentam o problema que os pesquisadores supunham ou cuja transferência não foi eficiente. Nesse sentido, Schlottfeldt (1991) afirma que, apesar do esforço despendido por profissionais de

\footnotetext{
* Universidade Tecnológica Federal do Paraná (UTFPR), Curitiba, PR, Brasil.

** Empresa Brasileira de Pesquisa Agropecuária (EMBRAPA), Concórdia, SC; Universidade Tecnológica Federal do Paraná (UTFPR), Curitiba, PR, Brasil.
} 
Transferência de Tecnologia (TT) e pesquisadores e do reconhecimento da necessidade de manter canais de comunicação de duas vias com os diversos públicos, permanece a visão de TT como mera disseminação de resultados da pesquisa.

Este trabalhou objetivou descrever e analisar o processo de comunicação científica e de TT da Empresa Brasileira de Pesquisa Agropecuária (Embrapa), a maior e principal instituição de pesquisa agropecuária brasileira. Trata-se de um estudo de caso constituído de três etapas: exploratória, com técnicas de revisão bibliográfica, análise documental e entrevistas semiestruturadas; descritiva, utilizando-se documentos impressos e eletrônicos, e documentos internos da Embrapa; e analítica, com dados obtidos por meio das entrevistas e da literatura consultada. $\mathrm{Na}$ Embrapa sede, foram entrevistados gerentes da Secretaria de Comunicação (SECOM), responsável pelo processo de comunicação, e gerentes do Departamento de Transferência de Tecnologia (DTT), responsável pelo processo de TT. Na Embrapa Suínos e Aves, foram entrevistados os empregados do Núcleo de Comunicação (NCO), responsável pelo processo de comunicação e empregados dos setores de Prospecção e Avaliação Tecnológica (SPAT) e Setor de Articulação e Implementação de Tecnologia (SPIT), responsáveis por atividades de TT. Os dados foram coletados a partir de entrevistas semiestruturadas no período entre janeiro a abril de 2013.

Este artigo está estruturado em cinco partes, além da parte introdutória: conceitos e definições sobre comunicação científica e transferência de tecnologia; contextualização sobre a comunicação científica e a TT em Instituições de C\&T; caracterização e atuação da Embrapa e seus processos de comunicação científica e TT; caracterização da Embrapa Suínos e Aves e seus processos de comunicação e de TT, finalizando com as conclusões.

\section{Comunicação científica e transferência de tecnologia: Conceitos e definições}

A comunicação científica é conceituada por autores clássicos como Garvey (1979) e Griffith (1989) como a comunicação que incorpora as atividades associadas à produção, disseminação e uso da informação, desde o momento em que o cientista concebe uma ideia até que a informação acerca dos resultados é aceita como constituinte do estoque universal de conhecimentos. De acordo com Chris Bryant, fundador do National Centre for the Public Awareness of Science, a comunicação científica é o processo pelo qual a cultura científica e seu conhecimento se incorporam à cultura comum (BRYANT, 2004, p. 1).

Cysne (1996, p. 8), aborda a transferência de tecnologia como sendo "a aquisição, entendimento, absorção e aplicação de uma tecnologia ou de um processo tecnológico".

Rogers e Shoemaker (1974) explicam que a diferença entre comunicação e transferência é que a primeira compreende todos os tipos de mensagens, enquanto a segunda se refere apenas a novas ideias. Ao contrário da comunicação, quando o receptor recebe mensagens rotineiras, na transferência a conduta deste é diferente porque envolve um grau de risco: aceitar ou rejeitar a novidade (ROGERS; SHOEMAKER, 1974, p. 12).

Segundo o National Technology Transfer Center (NTTC) (1999), existem três tipos principais de transferência de tecnologia:

- Spin-offTechnology: a tecnologia é desenvolvida por uma organização federal e transferida ao setor privado, a outra agência federal ou a governos locais.

- Spin-on Technology: refere-se às tecnologias viáveis comercialmente, desenvolvidas por organizações privadas, mas com potencial aplicação em organizações públicas.

- Dual-Use Technology: codesenvolvimento da tecnologia por uma organização pública e privada, com os custos divididos, e ambas são beneficiadas pela nova tecnologia.

A transferência de tecnologia também pode ocorrer de forma contratual. Os tipos de contratos mais comuns, segundo o Instituto Nacional da Propriedade Industrial (INPI) (2013) são:

- Licença para exploração de patente e desenho industrial: exploração por terceiros 
do objeto de patente ou desenho industrial, identificando direito de propriedade industrial.

- Licença para uso de Marca: autoriza o uso efetivo, por terceiros, de marca regularmente depositada ou registrada no país.

- Fornecimento de Tecnologia: estipula as condições para a aquisição de conhecimentos e de técnicas não amparados por direitos, incluindo conhecimentos e técnicas não amparados por propriedade industrial depositado ou concedidos no Brasil.

- Serviços de Assistência Técnica e Científica: obtenção de técnicas, métodos de planejamento e programação, pesquisas, estudos e projetos destinados à execução ou prestação de serviços especializados.

- Franquia: serviços, transferência de tecnologia e transmissão de padrões, além de uso de marca ou patente.

Rogers Takegami e Yin (2001) apresentam cinco mecanismos de transferência de tecnologia entre instituições públicas e a indústria:

- Contratos de Pesquisa e Desenvolvimento (P\&D), com compartilhamento de pessoal, custos e direitos intelectuais;

- Encontros de interação entre os pesquisadores, com troca de informação técnica;

- Publicações científicas - ressalvando que estas geralmente são direcionadas para parceiros científicos, e não aos usuários potenciais das tecnologias geradas;

- Contratos de licenciamentos;

- Spin-off acadêmico, em que a tecnologia será gerada por uma nova companhia, formada geralmente por ex-empregados da organização de origem.

\section{A comunicação científica e a transferência de tecnologia em Instituições de C\&T}

Para Oliveira (1998), é preciso defender veementemente a necessidade de divulgar $C \& T$ para justificar a pesquisa que é realizada, e por haver uma demanda reprimida de divulgação de C\&T no Brasil. Para a autora, uma das dificuldades que emperram o processo de comunicação em C\&T no Brasil é a ausência de uma cultura científica tanto nos profissionais da mídia, como no próprio público, que, na sua maioria, é incapaz de julgar criticamente ações e decisões políticas em C\&T, o que impede uma avaliação crítica das mensagens emitidas pelos gestores oficiais da C\&T. Segundo Geller (2010), é importante que a comunicação científica seja ampla, divulgando à sociedade as descobertas científicas que possam influenciar a vida das pessoas e, nesse processo, as instituições científicas desempenham importante papel, pois, além de gerarem conhecimento, podem contribuir para a popularização da ciência. Nesse contexto, Duran (2010) acredita que a comunicação científica é uma das ferramentas mais potentes para popularização da ciência e a apropriação de seus benefícios pela sociedade. Para Duarte (2004), as instituições públicas que atuam na área de C\&T são atores sociais mantidos pela sociedade que devem dar um retorno dos recursos públicos investidos, contribuindo para a evolução da sociedade, por meio da divulgação do conhecimento produzido e da inserção da C\&T no cotidiano das pessoas.

Entretanto Duarte (2004) ressalta que as pessoas que compõem as ICTs brasileiras vivem situações antagônicas na questão da comunicação, sendo responsáveis pela comunicação e democratização do conhecimento gerado e, ao mesmo tempo, pela criação de uma imagem pública que lhes garanta credibilidade e recursos. Para Araújo (1979), uma dificuldade para utilização bem sucedida da C\&T é a dificuldade de comunicação entre aqueles com o conhecimento do que é possível e aqueles que podem ser capazes de pôr esse conhecimento em uso, tornando-se necessário que os canais de comunicação e de transferência sejam ativos e funcionem de forma integrada. $\mathrm{O}$ autor, examinando os principais canais de comunicação para transferência, esclarece que livros, catálogos, folhetos técnicos, feiras técnicas, conferências, cursos de treinamento são responsáveis pelo despertar da atenção para avanços tecnológicos, mas não conduzem a uma efetiva TT uma vez que as capacidades necessárias para uma seleção e assimilação de tecnologia a partir destas fontes primárias são extremamente raras. Dereti (2009) ressalta ainda que é necessário diferenciar as ações e técnicas e instrumentos de comunicação que fazem parte do processo de TT, da transferência em si, pois esta não pode prescindir de ações de comunicação para atingir seus objetivos, mas ações de comunicação 
não caracterizam a TT. Na opinião de Schaun (1981), a TT é a consagração de toda a energia gasta para a geração do conhecimento e a validade da pesquisa, via adoção de tecnologia; daí por que se admite que, dentre os fatores limitantes à adoção das tecnologias geradas, está a forma de as transferir. Há ainda outro aspecto que deve ser considerado, que é a possibilidade de a tecnologia gerada não estar em consonância com a realidade do sistema social que se quer modificar, devido, principalmente, à falta de integração entre pesquisa-usuário (WILDNER et al., 1993). Nesse sentido, Fujisaka (1994) elenca uma série de razões pelas quais as tecnologias geradas não são adotadas, entre as quais, a de que a tecnologia resulta de um problema mal formulado pela pesquisa, ou seja, os usuários não enfrentam o problema que os pesquisadores supunham. Estudo realizado por Santoro \& Gopalakrishnan (2001) conclui que a confiança, a proximidade geográfica e a flexibilidade das políticas dos centros para os direitos de propriedade intelectual, de patentes e licenças têm um papel importante no sucesso das atividades de TT. O estudo confirmou que, quanto maior for a confiança entre as instituições de pesquisas e as empresas, a proximidade geográfica, a efetividade da comunicação e quanto mais flexíveis forem as políticas para os direitos de propriedade intelectual, de patentes e de licenças, maior será o grau de extensão das atividades de transferência de tecnologia.

Analisando a literatura citada, pode-se afirmar que os processos de comunicação científica e de TT estão interligados e, de certa forma, são interdependentes, pois o sucesso da transferência de uma tecnologia depende da eficiência dos canais e formas de comunicação, assim como um efetivo processo de TT pode gerar subsídios para melhorar tanto a comunicação científica como o processo de P\&D de uma instituição.

\section{Embrapa: Características e abrangência de atuação}

A Empresa Brasileira de Pesquisa Agropecuária (Embrapa) foi criada em 26 de abril de 1973, com as funções básicas de execução de pesquisa, desenvolvimento e inovação, e a transferência destas para o meio produtivo.
É uma empresa pública de direito privado, vinculada ao Ministério da Agricultura e do Abastecimento, constituindo-se na maior e principal instituição de pesquisa agropecuária brasileira, destacando-se na esfera internacional, como o principal centro de tecnologia agropecuária tropical do mundo. Atua por meio de 15 unidades administrativas e 47 Unidades de Pesquisa ou Serviço, presente em quase todos os Estados brasileiros, atuando também na América do Norte, Europa, Ásia, África e América Latina, por meio de Laboratórios Virtuais e projetos.

Coordena, ainda, o Sistema Nacional de Pesquisa Agropecuária (SNPA), constituído por instituições públicas federais, estaduais, universidades, empresas privadas e fundações que executam pesquisas nas diferentes áreas geográficas e campos do conhecimento científico. Seu quadro de pessoal é composto por 9.783 empregados (em 2012). Desse total, 2.389 são pesquisadores. Destes, $18 \%$ são mestres, $74 \%$ são doutores e $7 \%$ tem pósdoutorado, sendo que a maior parte deles está lotada nas Unidades Descentralizadas. O orçamento da Empresa em 2012 foi R\$ 2,3 bilhões.

\subsection{O processo de comunicação científica na Embrapa}

O processo de comunicação na Embrapa segue as diretrizes traçadas em sua Política de Comunicação, instituída em 1996, e é coordenado pela SECOM, Unidade Central da Embrapa. Para um melhor entendimento deste processo foram entrevistados dois gerentes lotados nessa secretaria.

A Embrapa trabalha com diferentes modalidades de comunicação, para atingir diferentes objetivos e públicos: comunicação científica, social, governamental, administrativa, mercadológica e comunicação para TT.

Conforme citado pelos entrevistados, as estratégias utilizadas partem das orientações da Política de Comunicação, estão voltadas para atender as vertentes P\&D, TT, Negócios e Desenvolvimento Institucional e variam de acordo com os objetivos e públicos a serem atingidos. Foram citados os seguintes instrumentos de comunicação: revista XXI; central de pautas para imprensa, articulação com a mídia; campanhas de promocionais 
de produtos/serviços; visitas de jornalista e demais formadores de opinião às Unidades; Programa Embrapa \& Escola, eventos como exposições, feiras, reuniões, seminários, dias de campo, cursos e programas de TV e rádio. Percebe-se que há um grande esforço em utilizar-se de mecanismos variados de comunicação, visando atingir diferentes públicos.

A formulação de estratégias de comunicação está centralizada na SECOM, que toma por base inicial a análise dos ambientes externo e interno e as demandas e orientações da direção da empresa. Pode-se dizer que contar com uma coordenação central é positivo para padronizar as ações nas unidades, porém, como cada unidade tem um público-alvo distinto, a participação destas na formulação das estratégias pode tornar o processo mais eficiente.

A SECOM realiza pesquisas de opinião, análise da presença da empresa na mídia, estudos de cenários, avaliação de eventos institucionais e técnicos. Percebe-se, porém, que essas pesquisas estão bastante voltadas para a comunicação institucional, havendo necessidade de incluir também a comunicação científica.

Os entrevistados acreditam que as estratégias e canais de comunicação utilizados atingem os diversos públicos-alvo, porém têm a percepção de que se devem melhorar seus canais de comunicação científica. Em relação às melhorias no processo, os entrevistados afirmam que a instituição está, constantemente, buscando melhorar sua comunicação, ressaltando, porém, a necessidade de melhorar as estratégias de comunicação científica com a criação de novos instrumentos e potencializar as ações nas redes sociais. Os entrevistados acreditam que houve um grande avanço no processo de comunicação, percebido pelo aumento do espaço dedicado à Embrapa tanto na imprensa quanto nos debates em fóruns públicos, o que resultou em um maior interesse da sociedade em assuntos de ciência e tecnologia.

Analisando o processo de comunicação da Embrapa, percebe-se que, apesar das políticas de comunicação implantadas, o processo de comunicação da instituição está mais fortemente voltado para a comunicação institucional, o que reforça a visão de Duarte (2004) de que as ICTs vivem um dilema na questão da comunicação, pois devem divulgar e democratizar o conhecimento e, ao mesmo tempo, criar uma imagem pública que lhes garanta credibilidade e recursos. No entanto, por se tratar de uma ICT pública, é imprescindível para a Embrapa buscar estratégias para ampliar e melhorar a comunicação científica, garantindo dessa forma sua legitimidade perante seus mais variados públicos.

\subsection{O processo de Transferência de Tecnolo- gia na Embrapa}

A TT na Embrapa visa disponibilizar os conhecimentos e as tecnologias geradas pela pesquisa aos diferentes segmentos da sociedade, por meio da articulação e integração entre as unidades de pesquisa, as unidades centrais e os parceiros nacionais e internacionais. Segundo o documento intitulado: Diretrizes para transferência de tecnologia: modelo de incubação de empresas (GOMES; ATRASAS, 2005), a Embrapa utiliza as seguintes modalidades de transferência:

Difusão de tecnologia: a apropriação pelo público-alvo é promovida por agentes de extensão rural, assistência técnica, redes de difusão, utilizando instrumentos como dias de campo, unidades de observação, cursos, palestras, publicações e mídias. Trata-se de conhecimentos isentos de proteção intelectual e;

Comercialização de tecnologias, que compreende:

- Licenciamento de uso: transferência da exploração comercial e uso da marca, mediante licitação e contrato, com remuneração pela transferência, pagamento de royalties sobre as vendas, assistência técnica e garantia de qualidade do produto comercializado.

- Alienação ou venda direta: transferência integral dos direitos de exploração da patente, mediante pagamento. Sujeita à licitação pública e regulamentação por contrato.

- Outras modalidades como prestação de serviços, consultorias e assessorias.

Os tipos de contratos mais utilizados nesse processo são os classificados de acordo com o INPI (2013), como Licença para exploração de patente; Fornecimento de Tecnologia e Serviços de Assistência Técnica e Científica.

Para entender melhor esse processo, foram entrevistados dois gerentes do 
Departamento de Transferência de Tecnologia (DTT), criado para sistematizar as estratégias prioritárias de TT junto às unidades, porém ainda não conseguiu atingir esse objetivo, devido ao fato de parte dos subprocesso de TT serem coordenados por outras unidades centrais. Assim, as estratégias de TT são desenvolvidas de forma descentralizada, diretamente pelas unidades de pesquisa, uma vez que a Embrapa não dispõe de uma política para orientar esse processo. Nesse aspecto, Eldred e McGrath (1997) afirmam que, para que a TT ocorra de forma eficaz, é fundamental a existência de um processo de gerenciamento estruturado, com uma clara definição de papéis e responsabilidade tanto para cada indivíduo envolvido quanto para o grupo em sua totalidade. Para os autores, o fracasso nos esforços de transferência deve-se, geralmente, não à incompetência dos indivíduos, mas sim à falta de metodologias claramente definidas e gerenciadas.

Os principais instrumentos utilizados para a TT são os cursos dias de campo, palestras, unidades demonstrativas e de observação, vitrines tecnológicas, eventos, planos de negócio, publicações técnicas, programas de rádio e TV e Minibibliotecas. Para Araújo (1979), esses instrumentos são mais eficientes no processo de comunicação, pois despertam o interesse do cidadão para os avanços tecnológicos, mas não conduzem a uma efetiva transferência e tecnologia.

Até o momento, não houve uma pesquisa ampla para medir a eficiência dos instrumentos e estratégias utilizados no processo de TT. O processo de TT não foi considerado eficiente pelos entrevistados, devido à forma como é feito (descentralizado) e sem uma política orientadora ou normas padronizadas, e pelo fato de poucas unidades de pesquisa possuírem capacidade técnica e estratégias para realizar ess e processo de forma eficiente. A falta de padronização das ações pode levar a ações desarticuladas entre as unidades e a sede, afetando a troca de informações e experiências, podendo fazer com que a Embrapa seja vista de maneira fragmentada pela sociedade.

Foram citadas, pelos entrevistados, as seguintes necessidades de melhorias deste processo:

- Elaboração de uma política norteadora de TT;
- Definição de uma governança interna para o processo;

- Redefinição de perfis profissionais (com instituição e capacitação das equipes);

- Mobilização de recursos financeiros e materiais para melhorar o processo nas unidades;

- Otimização da integração entre P\&D e TT e entre a Embrapa e as redes de TT;

- Fortalecimento das agências públicas e privadas de TT nos estados;

- Identificação das demandas das redes por tecnologias e ações de TT;

- Qualificação e organização do conhecimento e tecnologias por demandas;

- Identificação de melhores práticas e estratégias e TT;

- Avaliação dos impactos de tecnologias, estratégias, programação de P\&D e da evolução tecnológica em regiões e territórios.

Nota-se, pelas sugestões apresentadas, que o processo de TT ainda é bastante incipiente na Embrapa Sede, não havendo instrumentos e estratégias padronizadas ou normas que o regem, confirmando a percepção de Heberlê e Sapper (2006) de que, na Embrapa, não é clara a relação que envolve as etapas de geração e de transferência de tecnologia, o que pode tornar-se um ponto vulnerável para a instituição.

Partindo desse pressuposto, é importante para a Embrapa contar com um efetivo processo de TT para que possa reduzir o tempo entre a produção do conhecimento e das tecnologias e sua disponibilização junto aos usuários. Esse fato é reforçado por autores como Garnica e Torkomian (2009) e Schaun (1981), que ressaltam a necessidade das ICTs contarem com um processo eficaz de TT, para o alcance de um patamar tecnológico superior das empresas brasileiras. Sugere-se, também, a definição de uma governança para esse processo para que haja uma padronização, e de instrumentos e estratégias utilizados pelas unidades, pois, conforme Eldred e McGrath (1997), o sucesso do processo de transferência de tecnologia depende, em grande parte, de metodologias claramente definidas e gerenciadas. 


\section{A Embrapa Suínos e Aves}

A Embrapa Suínos e Aves é uma unidade de pesquisa da Embrapa e tem como missão "Viabilizar soluções de pesquisa, desenvolvimento e inovação para a sustentabilidade da suinocultura e avicultura em benefício da sociedade brasileira" (EMBRAPA..., 2011). Criada em 13 de junho de 1975, para pesquisar suínos, incorporando em1978 a pesquisa em aves, passando a se chamar Centro Nacional de Pesquisa de Suínos e Aves, hoje denominada Embrapa Suínos e Aves, localizada em Concórdia, SC, conta com um quadro de 211 empregados, sendo 51 analistas, 110 assistentes e 50 pesquisadores - destes, $86 \%$ são doutores e $14 \%$ são mestres.

4.1 O processo de comunicação científica na Embrapa Suínos e Aves

O processo de comunicação é coordenado pelo Núcleo de Comunicação Organizacional (NCO) e inicia-se na elaboração do projeto de pesquisa, que, obrigatoriamente, deve prever a forma de divulgação dos resultados, a qual é determinada pelo pesquisador responsável pelo projeto. Além de papers nacionais e internacionais, a unidade conta com publicações próprias, disponibilizadas em seu site, denominadas "Publicações da Série Embrapa", utilizadas na divulgação e difusão dos resultados das pesquisas realizadas. O tipo de publicação varia de acordo com o objetivo e público-alvo a ser atingido.

Para entender como ocorre o processo de comunicação científica, foram entrevistados os empregados que trabalham diretamente nesse processo, lotados no Núcleo de Comunicação Organizacional. Num total de seis empregados, cinco deles participaram da pesquisa realizada $(83,34 \%)$, respondendo aos questionários cujas questões versavam sobre o público-alvo das pesquisas da unidade, as estratégias e instrumentos de comunicação e possibilidades de melhorias nesse processo.

De forma geral, todos os entrevistados consideram como público-alvo da instituição a cadeia produtiva de aves, com seus diversos elos, também tendo sido citado o público formador de opinião, como imprensa e empresas do ramo do agronegócio.
As principais formas de interação com o público-alvo são os eventos, sendo também citadas notícias reproduzidas na mídia em geral, twiter, e as publicações disponibilizadas no site e em revistas científicas, sendo que estas mesmas formas foram citadas como principais instrumentos de comunicação científica, além de relatórios, publicações especializadas realeses, programas de rádio e TV, treinamentos, assessorias, dias de campo. Para os entrevistados, os instrumentos de interação com o público são os mesmos utilizados para publicação científica.

Em relação à formulação de estratégias de comunicação científica, não há uma percepção comum quanto à metodologia empregada. Foi citado que esta ocorre na elaboração do projeto de pesquisa; na elaboração do planejamento anual, de acordo com pesquisas, observações e até situações que precisam de respostas rápidas; que as estratégias são traçadas pelo NCO, por meio de contato direto com o pesquisador e por meio de projetos e elaborados para cada resultado de pesquisa consolidado e ainda por meio de contato com órgãos competentes. Não está claro para esses profissionais a forma como as estratégias de comunicação científica são traçadas, o que demonstra a falta de um planejamento estratégico. Quando perguntado se é feita pesquisa para avaliar a eficiência dos instrumentos de comunicação da unidade, três dos entrevistados citaram apenas a existência de avaliações pontuais de eventos realizados pela unidade; um entrevistado afirmou que a SECOM ou a unidade desenvolve ações desse tipo, que, mesmo pontuais, dão respostas interessantes, enquanto que outro acredita que não há esse tipo de pesquisa. Estas respostas levam a crer que a unidade não possui um instrumento para avaliar a eficiência de seu processo de comunicação científica. De forma geral, os entrevistados acreditam que as estratégias e canais de comunicação utilizados atingem o objetivo de comunicar C\&T, porém todos percebem a necessidade de melhorar a comunicação científica e criar novos instrumentos para isso. Essa percepção ficou clara ao ser indagado se a unidade dispõe de um banco de dados com os resultados das pesquisas realizadas. Todos responderam que estes se encontram disponíveis no site. Porém foi afirmado que essa disponibilização é bastante 
dispersa na Embrapa o que dificulta ao usuário acessá-las. Um entrevistado afirmou, ainda, que a unidade deve tentar fazer com que seus resultados de pesquisa se transformem em serviços e/ou produtos realmente utilizáveis no campo, a partir do recorte mais correto dos problemas de pesquisa. Observa-se, pelas respostas, que os resultados das pesquisas são disponibilizados para a sociedade, porém de forma ineficiente, pois o usuário não consegue localizar essas informações. Em relação à definição do público-alvo das pesquisas e a forma de comunicar os resultados, todos os entrevis- tados responderam que o projeto de pesquisa tem seu público-alvo definido, assim como a forma de comunicar os resultados obtidos. Todavia um entrevistado citou que, apesar terem um público-alvo definido, as pesquisas apresentam um problema de recorte do problema. Pelas respostas, percebe-se que há um esforço, por parte da equipe de comunicação, para divulgar os resultados das pesquisas, porém estes nem sempre trazem a resposta que o público-alvo necessita. Os entrevistados sugeriram algumas melhorias que poderiam tornar esse processo mais eficiente (Quadro 1).

\begin{tabular}{|l|c|}
\hline \multicolumn{1}{|c|}{ Melhoria sugerida } & $\begin{array}{c}\text { N. de entrevistados } \\
\text { que sugeriram }\end{array}$ \\
\hline Não há necessidade de melhorias & 1 \\
\hline Melhorar a disposição das informações no site & 2 \\
\hline Maior divulgação do site & 1 \\
\hline Fazer um planejamento da comunicação no momento de elaboração do projeto & 1 \\
\hline Melhorar o modelo de portfólios e arranjos da instituição & 1 \\
\hline Não sabe & 1 \\
\hline
\end{tabular}

Quadro 1 - Melhorias sugeridas pelos entrevistados para o processo de Comunicação da Embrapa Suínos e Aves.

Fonte: Dados de pesquisa (2013).

Nota-se que são sugeridas melhorias em diferentes etapas do processo, desde o planejamento da comunicação, até a disponibilização das informações para o usuário, sendo esta citada por mais de um entrevistado. Analisando o processo de comunicação da Embrapa Suínos e Aves, percebe-se que as atividades estão ainda bastante voltadas para a comunicação institucional, em detrimento da comunicação científica. As informações são disponibilizadas no site, porém de forma pouco eficiente, o que torna difícil sua localização pelo usuário. Além disso, o uso da mídia eletrônica não atinge os produtores rurais, pois a maioria destes não tem acesso à Internet, conforme demonstrado em pesquisa realizada por Bassi e Monticelli (2011). Observa-se ainda a falta de interação entre o pesquisador responsável pelo projeto e a equipe de comunicação, resultando em uma comunicação falha ou tardia dos resultados dos projetos.
4.2 O processo de transferência de tecnologia na Embrapa Suínos e Aves

Os instrumentos formais de transferência de tecnologia utilizados na Embrapa Suínos e Aves são os mesmos citados no documento: Diretrizes para transferência de tecnologia modelo de incubação de empresas (GOMES; ATRASAS 2005) ${ }^{1}$, ou seja, a difusão e comercialização de tecnologias, sendo mais comum neste caso, os contratos de licenciamento de uso para tecnologias geradas pela unidade ou em parceria e a prestação de serviços de consultoria e assessorias.

O processo de TT permeia por diversas áreas, mas está mais concentrado nos setores de Prospecção e Avaliação Tecnológica (SPAT) e pelo Setor de Articulação e Implementação de Tecnologia (SPIT). Assim, para um melhor entendimento desse processo, foram entrevistados os empregados que atuam

\footnotetext{
${ }^{1}$ Informações obtidas em conversa informal com um dos dirigentes da Unidade.
} 
nesses dois setores. Nesse caso, trata-se de 10 empregados, sendo que nove $(90 \%)$ participaram da pesquisa. As questões versavam sobre o público-alvo das pesquisas, as estratégias e instrumentos de TT e possibilidades de melhorias nesse processo.

As cadeias avícolas e suinícolas foram citadas como o público-alvo das pesquisas da unidade por oito dos nove entrevistados, sendo também citadas as instituições públicas de pesquisa, empresas de economia de mercado, grandes agroindústrias, instituições internacionais e alunos e técnicos de instituições públicas e privadas. Um dos entrevistados citou apenas que o público alvo deveria ser "o homem do campo somado a vertentes estratégicas de grandes arranjos definidas em âmbito governamental". Apesar de as cadeias avícolas e suinícolas serem consideradas o grande público-alvo da unidade, não há concordância em relação a qual segmento dessas cadeias é considerado prioritário para a instituição.

Quanto às formas de interação com esses públicos, as respostas foram as mais diversas. Foram citados eventos, SAC, projetos de P\&D, cursos, assessorias, contato direto com empresas, secretários de organizações governamentais, ministros e agentes de mercado. Também foi citado que ocorre somente após a demanda por parte dos interessados ou, ainda, por meio da disponibilização dos resultados das pesquisas para os públicos de interesse. Um entrevistado acredita que ocorre quando a agroindústria não consegue resolver um problema e procura a instituição para "trabalhar para ele", enquanto que, para outro entrevistado, há pouca interação com o público, exceto com os mais tecnificados e com acesso aos meios de comunicação digitais. Nota-se que, para os entrevistados, a interação ocorre não apenas pela disponibilização das informações, mas pela interação com o público. Porém a visão de como isso ocorre é bastante diversificada.

Em relação à estratégia e instrumentos de TT, as publicações tipo Embrapa, eventos internos e externos, cursos, livros, apostilas, maquetes, vídeos, banners, dias de campo, unidades demonstrativas foram citados por seis entrevistados, também tendo sido ressaltada a dificuldade em encontrar as informações no site. A participação em feira, para um dos entrevistados, é uma estratégia ineficaz. Dois entrevistados afirmaram que não há uma estratégia implantada, sendo que um deles citou que muitos resultados acabam em papers não lidos. Nesse aspecto, nota-se que, apesar de a maioria dos entrevistados citarem os eventos e publicações como principais instrumentos de TT, três (33\%) dos entrevistados acreditam que esses instrumentos são ineficazes, e outros dois (22\%) afirmaram não haver estratégias para a divulgação dos resultados das pesquisas da unidade.

Quanto à elaboração das estratégias de TT, também há diferentes percepções por parte dos membros das equipes. Dois entrevistados afirmam que depende do resultado da pesquisa e do público-alvo. Outro acredita que depende dos interesses governamentais e acesso ao público alvo. Para outros dois entrevistados, as estratégias são traçadas no próprio projeto de pesquisa e executadas pelas áreas responsáveis, enquanto que um afirma que estas são traçadas apenas no final dos projetos, quando o resultado já está disponível. Dois dos entrevistados não sabem informar. Percebe-se que as estratégias de TT não estão claramente definidas e, quando traçadas, são bem pontuais, direcionadas para projetos específicos e discutidas sem a participação dos profissionais de transferência.

Em relação ao uso de instrumentos para apurar a eficiência da TT, sete dos entrevistados afirmam que não há este tipo de pesquisa, enquanto que um acredita que é feito pela Embrapa Sede, e outro afirma que já foram realizados trabalhos, porém os resultados não são devidamente considerados pela direção da empresa.

Quando perguntado se as estratégias e canais e comunicação e TT utilizados atingem os objetivos, dois entrevistados acreditam que atingem o objetivo de comunicar C\&T, mas não de transferi-la. Outros três afirmam que atingem apenas em parte, havendo necessidade de uma maior efetividade no processo. Para dois entrevistados, não há estratégias voltadas para os produtores que não têm acesso aos meios eletrônicos, e não possuem o hábito de ler informações técnicas. Apenas um entrevistado acredita que as estratégias usadas atingem seus objetivos. Diante das respostas, pode-se afirmar que as estratégias, sob o ponto de vista dos profissionais de TT, 
devem ser revistas para que possam atingir os objetivos a que se propõem.

Quanto à disponibilização de um banco de dados com os resultados das pesquisas, todos os entrevistados citaram as publicações disponibilizadas no site da instituição, afirmando, porém, que estas são difíceis de serem encontradas e acessadas pelos usuários.

Em relação à definição do público-alvo das pesquisas e a forma de disponibilizar os resultados, três entrevistados afirmaram que o público-alvo é definido no momento de elaboração do projeto, porém as estratégias de comunicação e TT são feitas de maneira bastante simplória, pelo próprio pesquisador, enquanto que um acredita que tanto público quanto as estratégias de TT estejam contempladas no projeto de pesquisa. Ainda foi afirmado que não há definição de público- alvo, exceto quando as pesquisas atendem demanda da agroindústria, que se encarrega de comunicar a quem interessa. Outros dois entrevistados consideram que os projetos de pesquisa da unidade não têm estratégias de TT, sendo que um deles acredita que o pesquisador elabora os projetos baseado em suas experiências ou demandas pontuais. As respostas demonstram que o público-alvo é determinado pelo tipo de pesquisa realizado, o que é coerente coma natureza das atividades da Instituição. Também foi perguntado sobre as possíveis melhorias no processo e, apesar de se tratar de uma questão aberta, houve algumas melhorias que foram sugeridas por mais de um entrevistado. Assim, para um para um melhor entendimento, as melhorias sugeridas foram classificadas e estão sumariamente descritas no quadro 2 .

\begin{tabular}{|l|c|}
\hline \multicolumn{1}{|c|}{ Melhoria sugerida } & $\begin{array}{c}\text { N. de entrevistados } \\
\text { que sugeriram }\end{array}$ \\
\hline Desenvolver pesquisas voltadas para solução de problemas das cadeias & 2 \\
\hline Desenvolver tecnologias com aplicação prática & 2 \\
\hline Envolver as equipe de TT e Comunicação na elaboração dos projetos & 3 \\
\hline $\begin{array}{l}\text { Desenvolver instrumentos para medir a eficiência dos instrumentos de TT } \\
\text { utilizados }\end{array}$ & 1 \\
\hline Utilizar impressos (mala direta) para os diversos públicos & 1 \\
\hline Desenvolver um plano de TT em parcerias com agentes externos & 1 \\
\hline $\begin{array}{l}\text { Desenvolver ações de prospecção tecnológica para orientar os projetos de } \\
\text { pesquisa }\end{array}$ & 1 \\
\hline
\end{tabular}

Quadro 2 - Melhorias sugeridas pelos entrevistados para o processo de TT da Embrapa Suínos e Aves.

Fonte: Dados da pesquisa (2013)

As sugestões apresentadas pela equipe de TT estão voltadas para o desenvolvimento de pesquisas que geram resultados práticos para as cadeias nas quais a unidade está inserida. Em relação a isso, autores como Wildner et al., (1993); Fujisaka (1994) e Rosa Neto (2006) ressaltam que um dos problemas do processo de TT é que a tecnologia gerada resulta de um problema mal formulado pela pesquisa, ou seja, os usuários não enfrentam o problema que os pesquisadores supunham. Os autores reiteram a necessidade de estabelecer uma integração maior entre pesquisador-usuário para que haja um processo de comunicação de "mão dupla", para possibilitar a identificação de novas demandas que auxiliem a tomada de decisão, por parte da pesquisa, em relação à geração/adaptação de novas tecnologias.

\section{Conclusões}

As estratégias de comunicação da Embrapa, apesar de utilizar uma grande diversidade de instrumentos, ainda estão bastante voltadas para a comunicação institucional, havendo necessidade de criar novos modelos voltados para a comunicação científica, uma vez que se trata de uma instituição pública de C\&T.

O processo de comunicação na Embrapa Suínos e Aves segue a mesma estratégia da Embrapa Sede. Seus principais instrumentos 
de comunicação são publicações técnicas disponibilizadas no site e participação em eventos. Porém, por se tratar de uma instituição pública que tem como parte de seu públicoalvo os produtores rurais de acesso restrito a mídia eletrônica, sugere-se que sejam criados novos mecanismos de interação com seus diversos públicos, o que pode ser feito melhorando a comunicação entre a equipe de comunicação e o pesquisador responsável pelo projeto de pesquisa.

Em relação à transferência de tecnologia a nível estratégico, esse processo é bastante incipiente, não havendo padronização de estratégias e instrumentos e de uma instância coordenadora, o que pode vir a tornar esse processo menos efetivo. Na Embrapa Suínos e Aves, percebe-se também o processo de TT não é padronizado, que as estratégias são definidas, na maioria das vezes, pelo próprio pesquisador responsável pelo projeto. Sugere-se assim, que haja uma maior participação da equipe de TT na definição das estratégias e instrumentos a serem utilizados para transferir a tecnologia gerada, pois entende-se que essa participação poderá contribuir de forma decisiva para a correta definição das estratégias e instrumentos para a transferência de tecnologia.

Com base nas melhorias apresentadas, sugere-se também que seja criado um mecanismo para identificar as principais demandas de tecnologias das cadeias de aves e suínos, no desenvolvimento de soluções adequadas para responder a elas, uma vez que a existência de demanda para uma tecnologia ou serviço é condição necessária para a transferência. Por se tratar de uma ICT, o processo de transferência de tecnologia é parte integrante e essencial do processo de $\mathrm{P} \& \mathrm{D}$ e deve ser incorporado aos procedimentos adotados pelos técnicos da instituição, tanto na elaboração dos projetos quanto no processo de comunicação.

Práticas como diagnóstico das necessidades tecnológicas do público-alvo, abertura de canais de comunicação intersetoriais e com os diversos segmentos da sociedade, podem ser diretrizes metodológicas que venham tornar os processos de comunicação e transferência mais eficazes.

A transferência spin-off tecnology, apesar de apresentar um grau maior de liberdade de escolha por parte do pesquisador sobre o tipo de pesquisa e tecnologia a ser gerada, apresenta um menor grau de interação com os potenciais usuários da tecnologia e com o mercado. Isso pode resultar no desenvolvimento de tecnologias um menor grau de aplicação prática, conforme constatado por alguns entrevistados. O uso de transferência Dual-Use Technology poderá fazer com que as pesquisas resultem em tecnologias que possam trazer soluções para os problemas existentes em sua área de atuação.

\section{Referências}

ARAÚJO, V. M. R. H. Estudo dos canais informais de comunicação técnica: seu papel na transferência de tecnologia e na inovação tecnológica. Ciência da Informação, Rio de Janeiro, v. 8, n. 2, p. 79-100, 1979.

BASSI, Nádia S. S.; MONTICELLI, Cícero J. Canais de comunicação preferenciais dos suinocultores, avicultores e técnicos agropecuários no sul do Brasil. Cadernos de Ciência \& Tecnologia, Brasília, v. 28, n. 1, p. 279-303, jan./ abr. 2011. Disponível em: <http:/ / seer.sct.embrapa.br/ index.php/cct/article/view/12043/6608>. Acesso em: 10 abr. 2013.

BRYANT, Chris. National centre for the public awareness of science, 2004. Disponível em: <http:/ /info.anu.edu.au/ CPAS/Science_Communication/index.asp $>$. Acesso em: 02 jul. 2013.

CYSNE, Fátima P. Transferência de tecnologia e desenvolvimento. Ciência da Informação, Brasília, v. 25, n. 1, p. 26-35, jan./abr. 1996.

DERETI, Rogério M. Transferência e validação de tecnologias agropecuárias a partir de instituições de pesquisa. Desenvolvimento e Meio Ambiente, Curitiba, $\mathrm{n}$. 19, p. 29-40, jan./jun. 2009.

DUARTE, Jorge. Da divulgação científica à comunicação. Associação Brasileira de Jornalismo Científico, 2004. Disponível em: <htpp://www.abjc.org.br>. Acesso em: 28 jan. 2013.

DURAN, Jorge, E. Algunas consideraciones sobre el periodismo científico em America Latina. Periodismo y Comunicación Científica en América Latina. Estado actual y desafios. In: SEMINARIO INTERAMERICANO DE PERIODISMO Y COMUNICACIÓN CIENTÍFICA, 13-15 oct. 2010. Anais... Buenos Aires, 2010.

ELDRED, Emmet; MCGRATH, Michel E. Commercializing new technology. Research Technology Management, Arlington , v. 40, n. 3, p. 41-47, maio/jun. 1997.

EMBRAPA SUÍNOS E AVES, 2011. Disponível em: <http://www.cnpsa.embrapa.br>. Acesso em: 20 out. 2013.

FUJISAKA, Sam. Learning from six reasons why farmers do not adopt innovation sintended to improve sustainability of upland agriculture. Agricultural Systems, v. 46, n. 4, p. 409-425, 1994.

GARNICA, Leonardo A.; TORKOMIAN, Ana L. V. Gestão de tecnologia em universidades: uma análise do patenteamento e dos fatores de dificuldade e de apoio à transferência de tecnologia no Estado de São Paulo. Gest. Prod., São Carlos, v. 16, n. 4, p. 624-638, out./ dez. 2009. 
GARVEY, William D., LIN, Nan, NELSON, Carnot E. Communication in the physical and social sciences. In: GARVEY, W. D. Communication: the essence of science; facilitating information among librarians, scientists, engineers, and students. Oxford: Pergamon, 1979.332p. (Appendix I, p. 280-299).

GELLER, Bruno. Lasinstituciones científicas y La comunicación pública de La ciencia. Periodismo y Comunicación Científicaen América Latina. Estado actual y desafios. In: SEMINARIO INTERAMERICANO DE PERIODISMO Y COMUNICACIÓN CIENTÍFICA, 1315 oct. 2010. Anais... Buenos Aires, 2010.

GOMES, Guarany C.; ATRASAS, Ana L. Diretrizes para transferência de tecnologia: modelo de incubação de empresas. Embrapa InformaçãoTecnológica, Brasília, 36, p. , 2005.

GRIFFITH, Belver C. Understanding science: studies of communication and information. Communication Research, Newbury Park, v. 16, n. 5, p. 600-614, out. 1989.

HEBERLE, Antonio L. O.; SAPPER, Sadi M. Impasses entre midiatização e intercâmbio tecnológico na Embrapa. In: CONGRESSO BRASILEIRO DE CIÊNCIAS DA COMUNICAÇÃO, 29., 4-9 set. 2006. Anais... Brasília: Intercom, 2006.

INSTITUTO NACIONAL DA PROPRIEDADE INDUSTRIAL (INPI). Contrato de Tecnologia, 2013. Disponível em: <http://www.inpi.gov.br/portal/artigo/contrato_ de_tecnologia_1351692514525\#3>. Acesso em: 2 jul. 2013.

NATIONAL TECHNOLOGY TRANFER CENTER. How to transfer technology, 1999. Disponível em: <http:// www.nttc.edu/training/guide/secc00.html>. Acesso em: 05 jul. 2013.
OLIVEIRA, Fabiola I. Ciência e tecnologia na comunicação social de instituições governamentais. 197p. Tese (Doutorado em Ciência da Comunicação) - Universidade de São Paulo (ECA/ USP), 1998.

ROGERS, Everett M.; SHOEMAKER, Floyd. La comunicación de inovaciones. México: Herrero Hermanos, 1974, 385p.

ROGERS, Everett M.; TAKEGAMI, Shiro; YIN, Jing. Lessons learned about technology transfer. Technovation, Amsterdã, v. 21, n. 4, p. 253-261, dez. 2001.

ROSA NETO, Calixto. Principais demandas dos técnicos da extensão e de produtores rurais acerca do processo de inovação tecnológica de uma instituição de pesquisa agropecuária. Porto Velho: Embrapa Rondônia, 2006. 20p. (Série Documentos).

SANTORO, Michael D.; GOPALAKRISHNAN, Shanti. Relationship Dynamics between University Research Centers and Industrial Firms: their impact on technology transfer activities. Journal of Technology Transfer, New York, v. 26, n. 1-2, p. 163-171, jan. 2001.

SCHAUN, Nicolau M. Difusão de tecnologia no Centro Nacional de Milho e Sorgo. Sete Lagoas: EMBRAPACNPMS, 1981. 27p.

SCHLOTTFELDT, Carlos B. Difusão de tecnologia e extensão rural na EMBRAPA: reflexões conceituais e práticas. Cadernos de Ciência \& Tecnologia, Brasília, v. 8, n. 1-3, p. 98-112, jan./dez. 1991.

WILDNER, Leandro P.; NADAL, Raul de.; SILVESTRO, Milton. Metodologia para integrar a pesquisa, a extensão rural e o agricultor. Agropecuária Catarinense. Florianópolis, v. 6, n. 3, p. 37-47, set. 1993. 